\begin{tabular}{|c|}
\hline $\begin{array}{r}\text { PRAMANA } \\
\text { journal of } \\
\text { physics }\end{array}$ \\
\hline
\end{tabular}

\title{
Charting the invisible in phase space (Abstract only)
}

\author{
ALVARO de RUJULA \\ Physics Department, CERN, CH-1211, Genève 23, Switzerland \\ E-mail: alvaro.derejula@cern.ch
}

\begin{abstract}
One challenge in particle physics is that not all the momenta relevant to many processes are observable. Some particles are nearly invisible (neutrinos and hypothetical neutralinos), others escape undetected down the beam pipes of colliders. One faces the task of extracting the maximum information (e.g. on the mass of the unobserved particles) from a set of more unknowns than constraining energy-momentum conservation equations. We study the simplest realistic case of current interest: single- $W$ production at a hadron collider, followed by its leptonic decay. We derive and discuss the statistically-optimal 'singularity variable' relevant to the measurement of the $W$ mass. In spite of its simplicity, this process is fairly non-trivial and constitutes a good 'training' example for the scrutiny of phenomena involving invisible objects. Our graphical analysis of the phase space is akin to that of a Dalitz plot, extended to such processes.
\end{abstract}

Keywords. Identification of new particles; LHC; kinematics; quantum field theory.

PACS Nos 11.10.-z; 11.10.Cd; 11.10.Ef; 11.10.Gh; 11.10.Hi; 11.15.Bt; 11.55.Hx; 12.38.Cy 